\title{
Study of clusters and hypernuclei production with the NICA/MPD experiment
}

\author{
Viktar Kireyeu ${ }^{1}$ \\ JINR, VBLHEP \\ Joliot-Curie 6, 141980 Dubna, Moscow region, Russia \\ E-mail: skvitek@gmail.com
}

\begin{abstract}
Heavy-ion collisions provide the unique possibility to create and investigate hot and dense matter in the laboratory. At the initial stage of the reaction a QGP is formed, while the final stage is driven by the hadronization process and the formation of clusters. The capture of the produced hyperons by clusters of nucleons leads to the hypernuclei formation which is a very rare process at strangeness threshold energies.

We report on the first results on the dynamical modeling of cluster formation with the combined PHSD+SACA (Parton-Hadron-Strings dynamics + Simulated Annealing Clusterization Algorithm) model at Nuclotron and NICA energies. The clusters selection in SACA is realised by a simulated annealing procedure to obtain the most bound configuration of fragments and nucleons.
\end{abstract}

Based on present predictions of the combined model we study the possibility to detect such clusters and hypernuclei in the NICA/MPD detector.

XXII International Baldin Seminar on High Energy Physics Problems

15-20 September 2014

JINR, Dubna, Russia

${ }^{1}$ Speaker 


\section{Introduction}

It is important to have the robust modeling of hypernuclei and cluster formation in order to study the detector replica and to have the possibility to optimize the experimental setup for the best efficiency. Modeling of the clusters formation is a complicated problem, there are very few transport models that can provide it, this aspect is often oversimplified or even omitted.

\subsection{PHSD+SACA model}

The Parton-Hadron-String Dynamics (PHSD) [1] is a microscopic off-shell transport approach that describes the full evolution of a heavy-ion collision at relativistic energies from the initial hard scatterings and string formation through the dynamical deconfinement phase transition to the quark-gluon plasma as well as hadronization and to the subsequent interactions in the hadronic phase.

Simulated Annealing Clusterisation Algorithm (SACA) [2] is based on the search for nucleon configurations with a minimal binding energy: SACA takes randomly one nucleon and adds it to another fragmet until the most bound configuration will be found. It helps to produce fragments (clusters and hyernuclei) not only in "fireball", but also at spectators.

For our studies we use PHSD and SACA together as one model: PHSD produces hadrons and stops at a certain time, then SACA begins to make clusters and hypernuclei. It is very important to choose a good starting time for clusterisation algorithm. If you start too early you will get a huge amount of clusters, if you start too late after stabilisation of particles multiplicity, you will get a very few fragments.

On the left panel of the Fig.1 is particles multiplicity over PHSD evolution time. For our studies we chose starting time at $t=4,8,10,15,20$ and $30 \mathrm{fm} / \mathrm{c}$. On the right panel is the multiplicity of different kind of particles and fragments for selected times. After $15 \mathrm{fm} / \mathrm{c}$ multiplicity is almost stable. So we decided to choose $t=15 \mathrm{fm} / \mathrm{c}$ as a starting time for SACA simulations at $11.45 \mathrm{GeV}$.

Fig. 2 shows the comparison of PHSD+SACA predictions with AGS E-802 data [3] for central $\mathrm{Au}+\mathrm{Au}$ collisions at $11.45 \mathrm{GeV}$. This is $\mathrm{dN} / \mathrm{dy}$ distributions for protons (left panel) and deuterons (right panel). As one can see, model reproduces experimental data well.
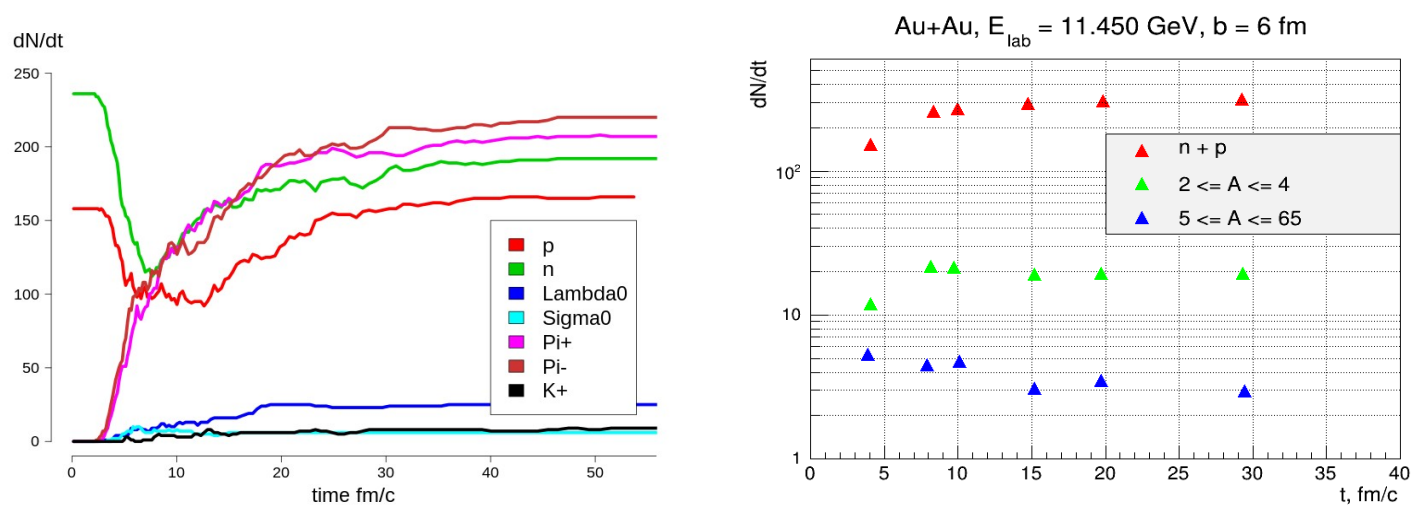

Figure 1: (left) Hadrons multiplicity over PHSD evolution time; (right) multiplicity of fragments over selected starting times. 

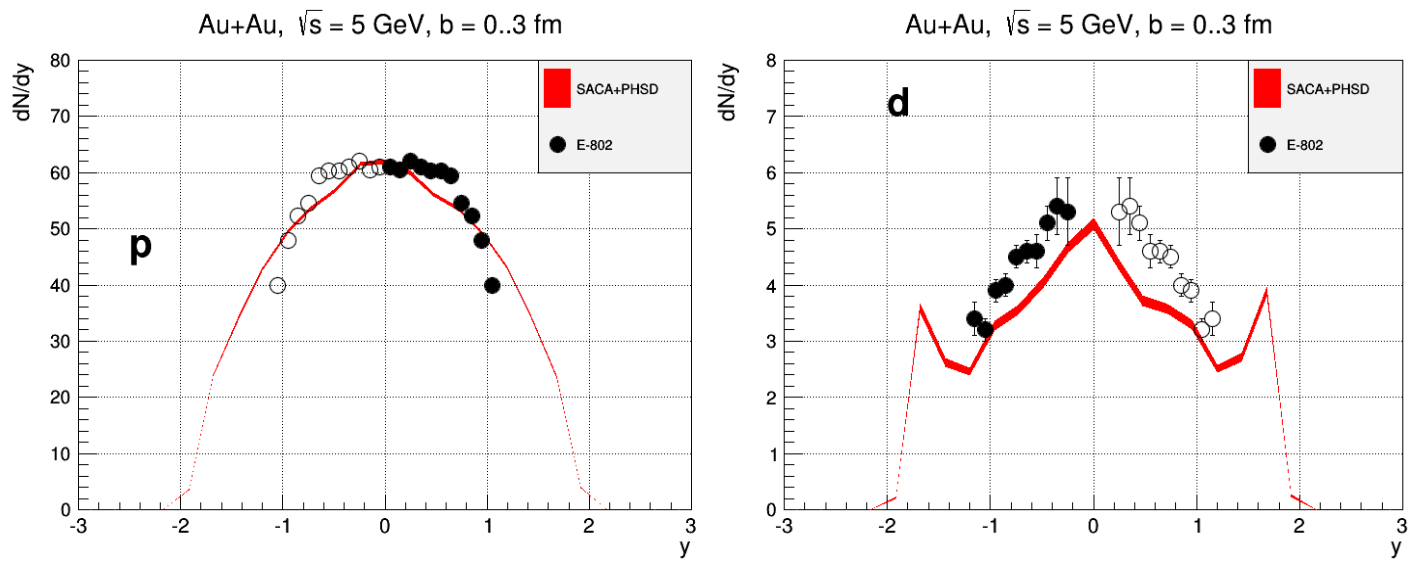

Figure 2: $d N /$ dy distributions for protons (left panel) and deuterons (right panel) in the central $A u+A u$ collisions at 11.45. Red lines is PHSD+SACA predictions, black filled circles is E-802 experimental data, black open circles is mirrored data.

\subsection{Multi-Purpose Detector feasibility study}

A new scientific program on heavy-ion physics NICA/MPD [4] was launched at JINR (Dubna) for comprehensive exploration of the QCD phase diagram with ion species ranging from protons to $\mathrm{Au}^{79}$ over the energy range $4<\sqrt{ } \mathrm{s}<11 \mathrm{GeV}$. The major goal of the NICA/MPD Project is the study of in-medium properties of hadrons and the nuclear matter equation of state, including a search for possible signals of deconfinement and/or chiral symmetry restoration phase transitions and the QCD critical endpoint.

One of the tasks of the Multi-Purpose Detector [5] is to study of the strangeness production. For this task is important to have a good identification and reconstruction of heavy strange objects like hypernuclei and hyperons.

Left panel of the Fig. 3 shows the yeilds of hypernuclei and hyperons of PHSD+SACA model for $\mathrm{Au}+\mathrm{Au}$ collisions at $11.45 \mathrm{GeV}$. Prelimenary results of the MPD feasibility study for hypertrinon are shown on the right panel. Hypertriton signal is clearly viewed, Signal-toBackground ratio is 3.3 , efficiency is about $0.4 \%$.
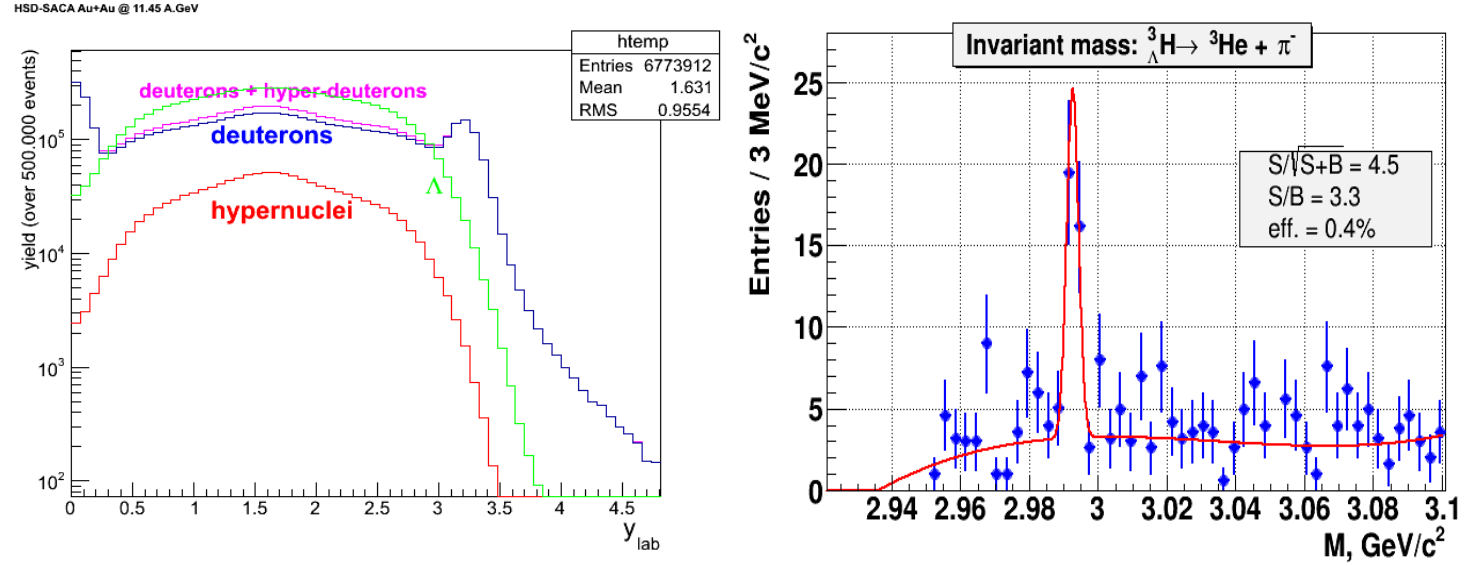

Figure 3: (Left) Yields of hypernuclei and hyperons in PHSD+SACA model, central $A u+A u$ collisions at $11.45 \mathrm{GeV}$; (right) Invariant mass distribution for He3 and $\pi$. 


\subsection{Conclusion}

PHSD+SACA model can produce clusters and hypernuclei and reproduce experimental data for $11.45 \mathrm{GeV}$, these predictions have been already used for MPD performance studies. Model is actively developing, there is some of polishing work to do.

\section{References}

[1] E.L. Bratkovskaya, W. Cassing, Nucl.Phys. A856 (2011) 162-182.

[2] R. K. Puri, J. Aichelin, J.Comput.Phys. 162 (2000) 245-266.

[3] «Proton and deuteron production in Au+Au reactions at 11.6A GeV/c» Phys. Rev. C, 60064901

[4] http://nica.jinr.ru/

[5] Kh.U. Abraamyan et al. (MPD Collaboration) NIM A 628 (2011) 99-102. 